\title{
ORIGINAL ARTICLE \\ The course of fatigue after acute spinal cord injury
}

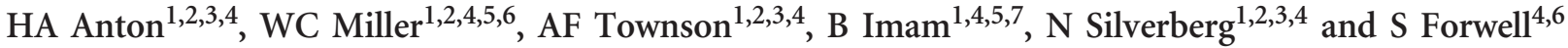

Study design: Prospective cohort study.

Objectives: To determine the prevalence and course of fatigue following acute spinal cord injury (SCl) during rehabilitation and after discharge.

Setting: Tertiary spinal cord rehabilitation facility.

Methods: Fifty-two patients with traumatic SCl were assessed after admission to rehabilitation and followed until 6-months post discharge into the community. Fatigue was measured using the Fatigue Severity Scale (FSS) and the Modified Fatigue Impact Scale for Spinal Cord Injury (MFIS-SCI) at admission, discharge and 6 months after discharge. Clinically significant fatigue was defined as FSS scores $\geqslant 4$ or MFIS-SCI scores $\geqslant 24.5$.

Results: The mean (s.d.) age of our mainly male (78.8\%) sample was 46.3 (17.8) years of age. Half had cervical and $61.6 \%$ had complete injuries. Mean (s.d.) FSS scores were 3.7 (1.6) at baseline, 3.4 (1.5) at discharge and 3.7 (1.7) post discharge. Mean (s.d.) MFIS-SCI scores were 24.4 (16.1) at baseline, 23.4 (16.3) at discharge and 27.8 (17.5) post discharge. Fatigue on the FSS was present in $51.9 \%$ at baseline, $38.3 \%$ at discharge and $48.1 \%$ post discharge. Fatigue on the MFIS-SCI was present in $44.2 \%$ at baseline, $44.7 \%$ at discharge and $51.9 \%$ post discharge. There was no relationship between fatigue and injury level or completeness. Conclusions: Fatigue is common in $\mathrm{SCl}$ patients admitted to rehabilitation. Fatigue remained stable during rehabilitation and after discharge into the community. Clinicians should consider early screening for fatigue and interventions to reduce the consequences of fatigue in people with $\mathrm{SCl}$.

Sponsorship: The British Columbia Medical Services Foundation funded our study.

Spinal Cord (2017) 55, 94-97; doi:10.1038/sc.2016.102; published online 28 June 2016

\section{INTRODUCTION}

Spinal cord injury (SCI) may cause severe neurological impairment and disability. It is frequently associated with secondary conditions that cause distressing symptoms and increased disability. Fatigue is a common secondary condition that occurs in over $50 \%$ of people with SCI living in the community. ${ }^{1,2}$ There is no single accepted definition of fatigue, but one useful definition is difficulty in initiating or sustaining voluntary activities. ${ }^{1}$ Fatigue has a negative association with participation in life activities after SCI. ${ }^{3}$ Fatigue may also have negative effects on involvement in rehabilitation after SCI. ${ }^{4}$ Use of mobility aids and wheelchairs may be adversely affected by fatigue., 5,6 Fatigue may contribute to excessive tiredness after performing cognitive tasks. ${ }^{7}$ Fatigue can also contribute to the reduction in health-related quality of life. ${ }^{8}$ Fatigue is an important concern to people living and aging with SCI in the community, and may contribute to decline in function and loss of independence. ${ }^{9-13}$ In summary, fatigue is an important problem in people with SCI, which can affect function, mobility and quality of life.

Many factors may contribute to fatigue after SCI. Potential causes of fatigue include medication use, depression, anemia, autonomic dysfunction, chronic pain, behavioral factors and sleep disorders. ${ }^{14-22}$
The increased energy costs associated with living and functioning with a physical impairment may also contribute to fatigue. ${ }^{23}$ Incomplete SCI was shown to contribute to increased fatigue severity in a sample of patients with SCI living in the community, possibly because those with incomplete injuries have less instrumental support and are more likely to participate in fatiguing activities. $^{2}$

Important knowledge gaps hinder the diagnosis, prevention and treatment of fatigue after SCI. Most research has focused on individuals with chronic SCI. There is little information about the course of fatigue in acute and subacute SCI. We are aware of only one study that assessed the prevalence of fatigue in the subacute stage after SCI. ${ }^{24}$ In this paper, we describe a prospective study that addresses the course of fatigue from initial rehabilitation to discharge into the community.

We hypothesized that fatigue will be common in people with acute and subacute SCI and will be more common in people with incomplete SCI. Because people with SCI are likely to have greater participation in fatiguing life activities after discharge to the community than in inpatient rehabilitation, we also hypothesized that fatigue will be greater in people with SCI after discharge.

${ }^{1}$ Rehabilitation Research Program, Vancouver Coastal Health Research Institute, Vancouver, BC, Canada; ${ }^{2}$ GF Strong Rehabilitation Centre, Vancouver Coastal Health Authority, Vancouver, BC, Canada; ${ }^{3}$ Division of Physical Medicine and Rehabilitation, Faculty of Medicine, University of British Columbia, Vancouver, BC, Canada; ${ }^{4}$ International Collaboration on Repair Discoveries, Vancouver, BC. Canada; ${ }^{5} \mathrm{GF}$ Strong Rehabilitation Research Lab, Vancouver, BC, Canada; ${ }^{6}$ Department of Occupational Sciences and Occupational Therapy, Faculty of Medicine, University of British Columbia, Vancouver, BC, Canada and ${ }^{7}$ Faculty of Medicine, Graduate Program in Rehabilitation Sciences, University of British Columbia, Vancouver, BC, Canada

Correspondence: Dr HA Anton, Division of Physical Medicine and Rehabilitation, University of British Columbia, Suite 140-943 West Broadway, Vancouver, BC V5Z 4E1, Canada. E-mail: hanton@pacificrehab.net

Received 23 December 2015; revised 15 April 2016; accepted 18 May 2016; published online 28 June 2016 


\section{MATERIALS AND METHODS}

\section{Design}

Our prospective cohort study aimed to recruit and follow individuals with new traumatic SCI from the time of admission to rehabilitation (the acute phase) to the community at 6-months post discharge (the subacute phase). Subjects were recruited from the G.F. Strong Rehabilitation Center in Vancouver, British Columbia, from 23 April 2008 to 30 September 2012. GF Strong is a tertiary referral center and the main provider of inpatient rehabilitation to people with traumatic SCI in British Columbia and the Yukon.

\section{Participants}

Previously inclusion criteria included age $\geqslant 19$ years of age and admission with acute traumatic SCI (paraplegia and tetraplegia, American Spinal Injury Association Impairment Scale (AIS) A, B, C and D) $<6$ months. ${ }^{25}$ Exclusion criteria included the inability to provide informed consent and anticipated discharge to a destination that would not allow follow-up. The Clinical Research Ethics Review Board of the University of British Columbia approved the study protocol. All applicable governmental and institutional regulations concerning the ethical use of human subjects were followed during the course of this research.

There were no previous studies that measured the incidence or prevalence of fatigue in the acute and subacute phase after SCI or in any similar population. We were therefore unable to perform an a priori power calculation for our study. We instead chose a sample size that was feasible within the time available to complete the study. We aimed for a sample size of 50 and planned to oversample by $10 \%$ to allow for subjects lost to follow-up. Enrollment and baseline data collection occurred within 1-4 weeks of admission to rehabilitation.

\section{Outcome measures}

Demographic and clinical variables collected included age, sex, marital status, education level, vocational status, days since injury, cause of injury, level of injury and completeness of injury. Information on medical comorbidities that might contribute to fatigue including other injuries, endocrine disorders, anemia, cardiac conditions, respiratory conditions, sleep disorders, additional neurological conditions and depression were obtained from review of the clinical charts. Fatigue data were collected during inpatient stay at baseline, at discharge and in the community at 6-months post discharge using telephone follow-up. Fatigue was measured using the Fatigue Severity Scale (FSS) and the Modified Fatigue Impact Scale for Spinal Cord Injury (MFIS-SCI). ${ }^{26-28}$

The FSS is a 9-item measure self-report tool for screening the severity of fatigue. ${ }^{26}$ Fatigue is rated on a 7-point ordinal scale ranging from 1 (strongly disagree) to 7 (strongly agree). Scores are calculated by deriving an arithmetic mean. Cut scores of over 4 are indicative of clinically significant fatigue. The FSS has been shown to be reliable and valid in people with SCI. ${ }^{2}$

The MFIS is a comprehensive multi-dimensional self-report scale that captures information regarding the impact of fatigue on an individual's life originally validated in people with multiple sclerosis. ${ }^{27}$ The MFIS has been adapted for the use in people with SCI (MFIS-SCI). ${ }^{28}$ Individuals rate their fatigue on a scale of 0 (never) to 4 (almost always). The scores are summed, yielding a total score of $0-84$, with higher scores indicating higher impact of fatigue. Cut scores of over 24.5 are indicative of clinically significant fatigue in people with SCI.

\section{Data analysis}

Variables were summarized as means (s.d.) or frequency (\%). We evaluated the temporal trends in fatigue by calculating the percentage of the sample that had clinically significant fatigue at baseline, at discharge and at 6-months post discharge (FSS scores $\geqslant 4$ or MFIS-SCI scores $\geqslant 24.5$ ). To further evaluate the effects of time from injury to the development of clinically significant fatigue, we compared the fatigue scores of patients with clinically significant fatigue at baseline with their scores at 6-months post discharge using odds ratios. If a $95 \%$ confidence of interval (CI) for the odds ratio does not include 1 , then the odds are said to be statistically significantly different. We also used linear regression modeling to look for the relationship between fatigue and level of injury (paraplegia or tetraplegia) and completeness of injury (motor complete AIS impairment A and B or motor incomplete AIS C and D).

\section{RESULTS}

We screened 179 individuals and recruited 67. We were able to collect complete data on 52 subjects (Figure 1). The demographic and clinical characteristics of our study subjects are contained in Table 1. Our sample had a mean (s.d.) age of 46.3 (17.8) years and was predominantly male $(78.8 \%)$. More than half $(61.6 \%)$ of our subjects had AIS A complete injuries. Half (50\%) had cervical level injuries (tetraplegia). Discharge data were available for only 39 of our 52 subjects because subjects were discharged prematurely before data could be collected or were transferred to acute care for medical treatment and then discharged directly home. Data were available from all 52 subjects at 6-months post discharge into the community.

Clinically significant fatigue was common in our sample, with approximately half having FSS or MFIS-SCI greater than the cutoff point (Tables 2 and 3). There was no statistically significant relationship between level or completeness of injury and fatigue $(P>0.05)$. There was no relationship between gender, age and fatigue.

The odds ratio (with associated 95\% CI) of having a clinically significant FSS score at 6-months post discharge was 2.59 times greater in those who had a clinically significant FSS at baseline than in those who did not $(95 \% \mathrm{CI}=0.86-8.20)$. On the basis of the $95 \% \mathrm{CI}$, the odds were not significantly different between the two groups on the FSS. The odds of having a clinically significant MFIS-SCI score at 6-months post discharge were 3.74 times greater in those who had a clinically significant MFIS-SCI at baseline than in those who did not $(95 \% \mathrm{CI}=1.21-12.57)$. On the basis of the $95 \% \mathrm{CI}$, the odds were significantly different between the two groups on the MFIS-SCI.

\section{DISCUSSION}

This study contributes to our knowledge of fatigue after SCI by providing data on fatigue in the acute stage of SCI. Previous studies have demonstrated that fatigue is common after chronic SCI and may occur in $52-57 \%$ of people living in the community. ${ }^{1,2,15}$ Our study shows that fatigue is also common in an acute and subacute

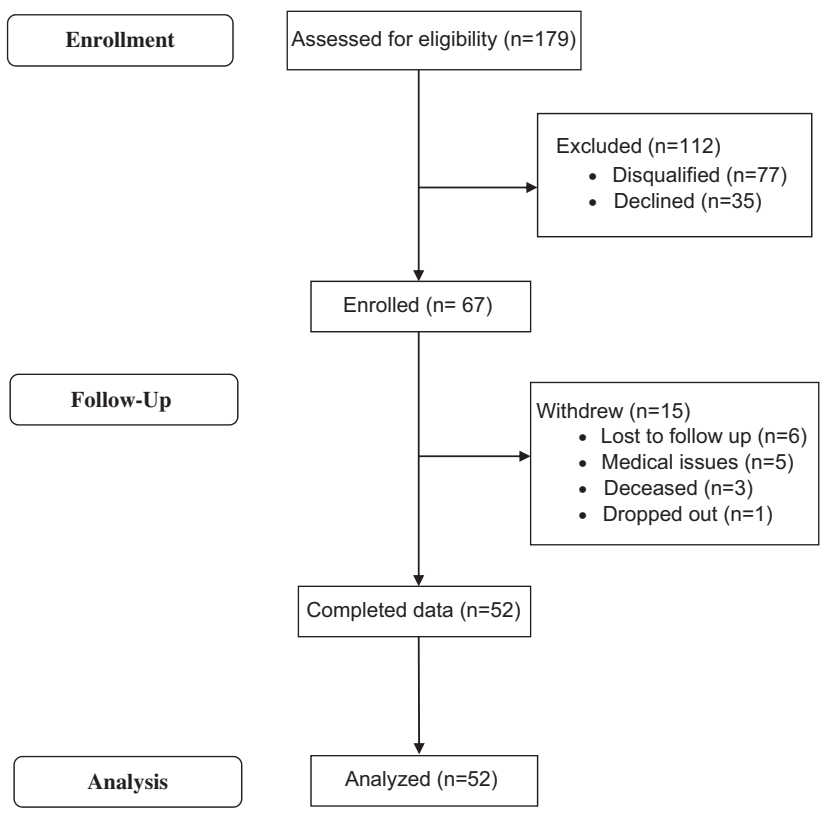

Figure 1 Participant flow diagram. 
Table 1 Summary of participant demographic and clinical characteristics $(n=52)$

\begin{tabular}{|c|c|}
\hline Age mean (s.d.) & $46.3(17.8)$ \\
\hline Male (\%) & $41(78.8)$ \\
\hline \multicolumn{2}{|l|}{ Marital status, $n(\%)$} \\
\hline Married & $19(36.5)$ \\
\hline Single & $17(32.7)$ \\
\hline Separated and divorced & $6(11.5)$ \\
\hline Common law & $9(17.3)$ \\
\hline Widowed & $1(1.9)$ \\
\hline \multicolumn{2}{|l|}{ Education level, $n$ (\%) } \\
\hline Less than high school & 2 (3.8) \\
\hline High school & $28(53.8)$ \\
\hline Some college & $8(15.4)$ \\
\hline University degree & $11(21.2)$ \\
\hline \multicolumn{2}{|l|}{ Vocational status, $n(\%)$} \\
\hline Retired & $7(13.5)$ \\
\hline Unemployed & $5(9.6)$ \\
\hline Employed & $38(73.1)$ \\
\hline Length (days) of stay mean (s.d.) & $110.8(51.1)$ \\
\hline Day since injury mean (s.d.) & $45.6(22.1)$ \\
\hline \multicolumn{2}{|l|}{ Completeness of injury, $n$ (\%) } \\
\hline Complete (AIS A and B) & $32(61.6)$ \\
\hline Incomplete (AIS C and D) & $20(38.5)$ \\
\hline \multicolumn{2}{|l|}{ Level of injury, $n(\%)$} \\
\hline Cervical & $26(50)$ \\
\hline Thoracic & $22(42.3)$ \\
\hline Lumbosacral & $4(7.7)$ \\
\hline
\end{tabular}

Abbreviation: AIS, AIS Impairment Scale.

Table 2 Frequency of clinically significant fatigue

\begin{tabular}{lccc}
\hline & Baseline, n (\%) & Discharge, n (\%) & Post 6 months, n (\%) \\
\hline FSS score $\geqslant 4$ & $27(51.9 \%)$ & $18(38.3 \%)$ & $25(48.1 \%)$ \\
MFIS-SCI score $\geqslant 24.5$ & $23(44.2 \%)$ & $21(44.7 \%)$ & $27(51.9 \%)$ \\
\hline
\end{tabular}

Abbreviations: FSS, Fatigue Severity Scale; MFIS-SCI, Modified Fatigue Impact Scale for Spinal Cord Injury.

Table 3 Change in fatigue over time

\begin{tabular}{ll}
\hline FSS mean (s.d.) & \\
Baseline & $3.7(1.6)$ \\
Discharge & $3.4(1.5)$ \\
6-Months post discharge & $3.7(1.7)$ \\
& \\
MFIS-SCI mean (s.d.) & \\
Baseline & $24.2(16.1)$ \\
Discharge & $23.4(16.3)$ \\
6-Months post discharge & $27.8(17.5)$ \\
\hline
\end{tabular}

Abbreviations: FSS, Fatigue Severity Scale; MFIS-SCI, Modified Fatigue Impact Scale for Spinal Cord Injury. population of individuals with recent SCI, occurring in nearly half of our study participants. In the only other study we are aware of that measured the prevalence of fatigue in subacute SCI, Nooijen et al. ${ }^{24}$ found fatigue in only $31 \%$ of their sample. Their study had a smaller sample size and included people with both traumatic and nontraumatic SCI. It is possible that people with non-traumatic SCI have different patterns of neurological injury and impairment, which might reduce the prevalence of fatigue.

We hypothesized that patients with incomplete SCI in our study would be more likely to have fatigue. Our results did not support that hypothesis. That contrasts with the results of Nooijen and the FawkesKirby group, who did find that fatigue was more common in people with incomplete SCI. One possible explanation is that our study participants were somehow different than those in the other studies. The Nooijen and Fawkes-Kirby studies included some participants with non-traumatic SCI, but it is not clear why that might explain their different results. Another possible explanation is that time as injury has a different effect on fatigue in incomplete versus complete SCI. The mean time from injury to initial assessment in our study was 45.6 days. The time to assessment after discharge in the community varied greatly, but all the data collection was completed within 1 year from the time of injury. The mean duration of time since injury in the study by Nooijen's group was 4.7 months. The mean duration since injury in the study by Fawkes-Kirby and colleagues was 12.68 years. Given that the time between injury and fatigue assessment in our group falls between that in the Nooijen and Fawkes-Kirby studies, it does not seem that time since injury would explain the difference in our results.

Level of injury is another factor that could contribute to fatigue after SCI. People with lower level injuries are generally more able to participate in physical activities than people with higher level injuries. That increased participation could contribute to increased fatigue. Alternatively, it has been suggested that decreased sympathetic activity in people with tetraplegia compared with those with paraplegia could contribute to increased fatigue. ${ }^{18}$ There was no relationship between the level of injury and fatigue in our study. Given our relatively small study sample and the heterogeneous nature of injury characteristics based on the injury level, it may be that our study did not have the power to identify such a relationship.

Medical comorbidities such as disordered sleep, anemia, orthostatic hypotension or pain are common in people with SCI and may contribute to fatigue. ${ }^{13,14,16,17,19}$ We collected data on medical comorbidities from our subjects' medical charts. Medical conditions were common in our study population, but we had no way of assessing their severity, current clinical relevance or relationship to fatigue.

People with SCI frequently use sedating medications such as antispasticity drugs, analgesics for neuropathic or nociceptive pain and antidepressants. Medication use has been associated with fatigue in patients with chronic SCI. ${ }^{14}$ We previously assessed the effect of inpatient medication use in a subset of our study population and reported the results elsewhere. ${ }^{29}$ The most prescribed medication category was analgesics. Analyses showed no significant relationship between specific medication classifications or the number of medications and fatigue scores. It is possible that the sample size was too small to identify any clinically significant effects of medication on fatigue in our population.

We used two different fatigue measures in our study. We did so in part because fatigue is a complex phenomenon, and the FSS and MFIS-SCI measure different aspects of fatigue. Use of both the FSS and MFIS-SCI increases the relevance of our study to clinicians. Our 
experience suggests that clinicians can be comfortable using either tool based on their specific needs. The FSS is a better screening tool because it is simple and takes only a very short time to administer. The more comprehensive MFIS-SCI is better suited to measure the effects of rehabilitation treatment.

To the best of our knowledge, ours is the only study that has prospectively followed patients with acute SCI from the start of rehabilitation to discharge and then to the community. We hypothesized that fatigue will be greater in people with SCI after discharge. Our results for the most part did not support that conclusion. We found that the odds of having a clinically significant MFIS-SCI score at 6-months post discharge were significantly greater in those who had a clinically significant MFIS-SCI at baseline than in those who did not, but did not find a similar result with the FSS. One explanation for the mixed results is that our numbers were too small to show a significant change with the FSS. It is also possible that there is more than one trajectory for fatigue after injury and significant individual differences in the course of fatigue were not fully captured in our study. The most conservative interpretation of our results is that fatigue is stable over the course of time from admission to the completion of rehabilitation and after discharge into the community. An important clinical implication is that if fatigue is identified early after injury then fatigue is likely to persist.

We encourage clinicians to consider measuring fatigue with a tool similar to the FSS or the MFIS-SCI after admission to rehabilitation. If fatigue is present, the next step should be to identify and treat potentially reversible causes of fatigue, such as depression, use of sedating medications and comorbid medical conditions. Clinicians should also implement strategies for fatigue management throughout the rehabilitation process to reduce the long-term consequences of fatigue for their patients with SCI.

\section{Study limitations}

The most important limitation in our study was our relatively small sample size. Another limitation was that we were not able to follow subjects for a longer period after discharge into the community. Although we collected data on medical comorbidities, we had insufficient information to evaluate the potentially important contribution of those to fatigue. Finally, because fatigue is a complex phenomenon, it is possible the fatigue measures we used may not have fully captured all aspects of fatigue.

\section{DATA ARCHIVING}

There were no data to deposit.

\section{CONFLICT OF INTEREST}

The authors declare no conflict of interest.

\section{ACKNOWLEDGEMENTS}

We acknowledge the support of the British Columbia Medical Services Foundation, the Vancouver Coastal Health Research Institute and the Rehabilitation Research Program at the GF Strong Rehabilitation Centre. Funding for this project was provided by the British Columbia Medical Services Foundation. Ms BM is supported by the Vanier Canada Graduate Scholarships. Dr NS receives salary support from the Vancouver Coastal Health Research Institute.
1 Anton HA, Townson AF, Miller WC. Measuring fatigue in persons with spinal cord injury. Arch Phys Med Rehabil 2008; 89: 538-542.

2 Fawkes-Kirby T, Wheeler M, Anton HA, Miller WC, Townson AF. Clinical correlates of fatigue in spinal cord injury. Spinal Cord 2008; 46: 21-25.

3 Smith EM, Imam B, Miller WC, Silverberg ND, Anton HA, Forwell SJ et al. The relationship between fatigue and participation in spinal cord injury. Spinal Cord 2015; 54: 457-462.

4 Hammond FM, Lieberman J, Smout RJ, Horn SD, Dijkers MP, Backus D. Missed therapy time during inpatient rehabilitation for spinal cord injury. Arch Phys Med Rehabil 2013; 94: S106-S114.

5 Dipiro ND, Saunders LL, Brotherton S, Kraft S, Krause JS. Pain and fatigue as mediators of the relationship between mobility aid usage and depressive symptomatology in ambulatory individuals with SCl. Spinal Cord 2014; 52: 316-321.

6 Saunders LL, Krause JS, DiPiro ND, Kraft S, Brotherton S. Ambulation and complications related to assistive devices after spinal cord injury. J Spinal Cord Med 2013; 36: 652-659.

7 Craig A, Tran Y, Wijesuriya N, Middleton J. Fatigue and tiredness in people with spinal cord injury. J Psychosom Res 2012; 73: 205-210.

8 Wijesuriya N, Tran Y, Middleton J, Craig A. Impact of fatigue on the health-related quality of life in persons with spinal cord injury. Arch Phys Med Rehabil 2012; 93: 319-324.

9 McColl MA, Arnold R, Charlifue S, Glass C, Savic G, Frankel H. Aging, spinal cord injury, and quality of life: structural relationships. Arch Phys Med Rehabil 2003; 84: 1137-1144.

10 Gerhart KA, Bergstrom E, Charlifue SW, Menter RR, Whiteneck GG. Long-term spinal cord injury: functional changes over time. Arch Phys Med Rehabil 1993; 74: 1030-1034.

11 Charlifue SW, Weitzenkamp DA, Whiteneck GG. Longitudinal outcomes in spinal cord injury: aging, secondary conditions, and well-being. Arch Phys Med Rehabil 1999; 80: 1429-1434.

12 Thompson L, Yakura J. Aging related functional changes in persons with spinal cord injury. Top Spinal Cord Injur Rehabil 2001; 6: 69-82.

13 Alschuler KN, Jensen MP, Sullivan-Singh SJ, Borson S, Smith AE, Molton IR. The association of age, pain, and fatigue with physical functioning and depressive symptoms in persons with spinal cord injury. J Spinal Cord Med 2013; 36: 483-491.

14 Lee AKY, Miller WC, Townson AF, Anton HA. Medication use is associated with fatigue in a sample of community-living individuals who have a spinal cord injury: a chart review. Spinal Cord 2010; 48: 429-433.

15 Lidal IB, Jensen AE, Larsen TW, Stanghelle JK. Fatigue in persons who have lived with spinal cord injury for $>20$ years. Spinal Cord 2013; 51: 103-108.

16 Hirsch GH, Menard MM, Anton HA. Anemia in spinal cord injury. Arch Phys Med Rehabil 1991; 72: 195-201.

17 Streeten $\mathrm{DH}$, Anderson $\mathrm{GH}$. The role of delayed orthostatic hypotension in the pathogenesis of chronic fatigue. Clin Auton Res 1998; 8: 119-124.

18 Rodrigues D, Tran Y, Guest R, Middleton J, Craig A. Influence of neurological lesion level on heart rate variability and fatigue in adults with spinal cord injury. Spinal Cord 2015; 54: 292-297.

19 Widerstrom-Noga EG, Felipe-Cuervo E, Yezierski RP. Chronic pain after spinal cord injury: Interference with sleep and daily activities. Arch Phys Med Rehabil 2001; 82: 1571-1577.

20 Craig A, Tran Y, Siddall P, Wijesuriya N, Lovas J, Bartrop R et al. Developing a model of associations between chronic pain, depressive mood, chronic fatigue, and self-efficacy in people with spinal cord injury. J Pain 2013; 14: 911-920.

21 Saunders LL, Krause JS. Behavioral factors related to fatigue among persons with spina cord injury. Arch Phys Med Rehabil 2012; 93: 313-318.

22 Giannoccaro MP, Kaveh Moghadam KK, Pizza F, Boriani S, Maraldi NM, Avoni P et al. Sleep disorders in patients with spinal cord injury. Sleep Med Rev 2013; 17: 399-409.

23 Weingarden SI, Martin C. Independent dressing after spinal cord injury: a functional time evaluation. Arch Phys Med Rehabil 1989; 70: 518-519.

24 Nooijen CFJ, Vogels S, Bongers-Janssen HMH, Bergen MP, Stam HJ, van den Berg-Emons HJG et al. Fatigue in persons with subacute spinal cord injury who are dependent on a manual wheelchair. Spinal Cord 2015; 53: 758-762.

25 Committee Membership, Burns S, Biering-Sørensen F, Donovan W, Graves DE, Jha A et al. International standards for the neurological classification of spinal cord injury. Revised 2011. Top Spinal Cord Inj Rehabil 2012; 18: 85-99.

26 Krupp LB, LaRocca NG, Muir-Nash J, Steinberg AD. The Fatigue severity scale: application to patents with multiple sclerosis and systemic lupus erythematosus. Arch Neurol 1989; 46: 1121-1123.

27 Kos D, Nagels G, D'Hooghe MB, Duportail M, Kerckhofs E. A rapid screening tool for fatigue impact in multiple sclerosis. BMC Neurol 2006; 6: 27.

28 Imam B, Anton HA, Miller WC. Measurement properties of a telephone version of the Modified Fatigue Impact Scale among individuals with a traumatic spinal cord injury. Spinal Cord 2012; 50: 920-924.

29 Imam B, Townson A, Anton H, Miller W, Forwell S. Medication use and fatigue in individuals with an acute spinal cord injury. Top Spinal Cord Inj Rehabil 2014; 20: S16. 